\title{
DESIGN AND FABRICATION OF POSFET DEVICES FOR TACTILE SENSING
}

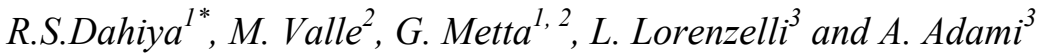 \\ ${ }^{1}$ Italian Institute of Technology, 16163, Genoa, ITALY \\ ${ }^{2}$ University of Genoa, 16145, Genoa, ITALY \\ ${ }^{3}$ Fondazione Bruno Kessler, 38050, Trento, ITALY
}

\begin{abstract}
This work presents the design and fabrication of novel piezoelectric polymer-FET touch sensing devices. With thin piezoelectric polymer (PVDF-TrFE) film directly spun and processed in situ on the gate area of MOS transistor, the device can also be called as POSFET (Piezoelectric Oxide Semiconductor Field Effect Transistor) touch sensing device. The integral POSFET "sensotronics" unit, comprising of transducer and the transistor, senses and conditions the signal at 'same site'. Challenging issues like in situ poling of piezoelectric polymer film, without damaging or altering the characteristics of underlying MOS devices, have been successfully dealt with.
\end{abstract}

\section{KEYWORDS}

POSFET, Touch sensor, Tactile sensing, Piezoelectric polymer-FET, Sensotronics.

\section{INTRODUCTION}

Touch sensing plays an important role in various application domains like robotics, electrotextiles and medical prosthesis, as it facilitates the detection and measurement of rich interaction behaviors (e.g. how heavy and hard an object is when hold, how its surface feels when touched, how it deforms on contact and how it moves when pushed etc.) exhibited by real world objects [1]. Over the years, touch sensing technology has improved and many new touch sensors, using different materials and transduction methods, viz: Resistive/Piezoresistive, Quantum Tunneling, Capacitive, Optical, Ultrasonic, Magnetic, Piezoelectric etc. have been developed [2]. Many of these sensors are, in general, big in size and thus unsuitable for body sites like robot's fingertips where large numbers of sensors with high density are needed. Miniaturized touch sensor, using MEMS approach, have also been developed [3, 4]. However, due to fragile nature, such sensors are not even able to cover the range of force experienced by humans in a normal manipulative task. Thus usage of such sensors is limited to applications where very small contact forces are to be measured. Mechanically flexible touch sensors using organic FETs have also been developed for large area skin type of applications [5, 6]. However, best organics are known to have a mobility of about $1 \mathrm{~cm}^{2} / V s$ versus 85 $\mathrm{cm}^{2} / V s$ for silicon based MOS devices [7], which limits their usage to recording of slow varying contact forces only.

This work presents the design and fabrication of novel POSFET touch sensing devices, primarily developed for the fingertips of robotic hand - where large number of sensors with high density is needed. POSFET touch sensing devices are fabricated by spin coating thin $(\sim 2.5$ $\mu m$ ) film of piezoelectric polymer, PVDF-TrFE, directly on to the gate area of MOS transistor. The POSFET device, thus, represents an integral "sensotronics" unit, which is capable of sensing and conditioning the touch signals at 'same site'. The POSFET approach brings the sensor and conditioning electronics closer and hence the overall response is better than that of conventional approach - in which, the sensor and conditioning electronics are placed at a distance. The marriage of sensing material and the electronics also improves signal to noise ratio and hence the force sensitivity. The POSFET touch sensors, presented here, are designed to match the cutaneous/tactile sensing of human fingertips.

This paper is organized as follows: The concept of POSFET touch sensing device is presented in section II. Section III presents the design and the fabrication of POSFET touch sensing devices.

\section{THE CONCEPT OF POSFET DEVICE}

A piezoelectric polymer film, in the sensing mode, generates a charge/voltage on application of a mechanical force/stress [8], which is approximately given as:

$$
Q_{t}=d_{33} \times F
$$

Where, $Q_{t}$ is the charge developed due to applied force $F$ and $d_{33}$ is the piezoelectric constant. With piezoelectric polymer film on the gate area of MOS device, as shown in Fig. 1, such a charge/voltage modulates the charge/current in the induced channel of MOS device. Thus, POSFET devices directly convert mechanical stimulus (force, in present case) into voltage - which can be amplified by POSFET itself and further processed by an electronic circuitry that may be present on the same chip. In this sense, POSFET touch sensing device can also be compared with the mechanoreceptors - the receptors that sense the contact parameters in the human skin - and convert them into partially processed electrical signals [9]. 


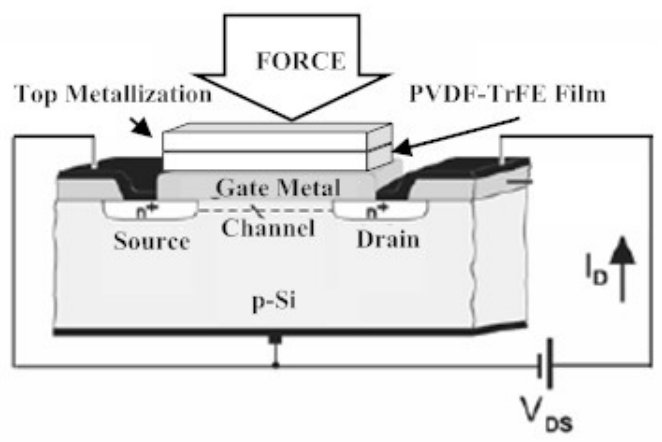

Figure 1: The concept of POSFET touch sensing device.

\section{DESIGN AND FABRICATION}

The POSFET touch sensing device, implemented on silicon substrate is shown in Fig. 2. In order to match the spatial acuity of receptors in human skin, POSFET touch sensing devices are designed to have size of $1 \mathrm{~mm} \times 1 \mathrm{~mm}$. In fact, better spatial acuity can be obtained by reducing the size of transistor. At fingertips, humans are able to differentiate two points as close as $1 \mathrm{~mm}$, with similar spatial acuity. The properties like spatial resolution can be easily modified, as per the requirements of an application, by changing the size of MOS device. To have large transconductance, the n-MOS devices are designed to have $\mathrm{W}=7500 \mu \mathrm{m}$ and $\mathrm{L}=12 \mu \mathrm{m}$ and an aspect ratio of about 600 is thus obtained. $\mathrm{A} \mathrm{Si}_{3} \mathrm{~N}_{4} / \mathrm{SiO}_{2}$ double layer is used as a gate dielectric.

The fabrication steps for developing POSFET tactile sensors are shown in Fig. 3. For first six steps of Fig. 3, the fabrication steps are similar to that of an n-MOS technological module of a non standard 4- $\mu m \mathrm{Al}$ gate $\mathrm{p}$ well ISFET/CMOS technology [10]. The fabrication steps 7-8 are related with the deposition and in situ processing of the piezoelectric polymer film on the gate area of underlying MOS devices. The polymer related steps, explained in following sections, include spin coating of polymer film; annealing; top metallization; dry etching and poling of polymer films.

\section{Selection of the polymer film material}

PVDF and its copolymer, PVDF-TrFE, are wellknown polymers widely used in various piezoelectric polymers based sensors due to excellent features like, flexibility, workability, and fast dynamic response. PVDFTrFE, a copolymer of PVDF, has the tendency to crystallize directly in the polar -phase without any requirement of mechanical stretching. PVDF, on the other hand, requires mechanical stretching to transform from the nonpolar -phase to the polar -phase. In other words, mechanical stretching is needed to induce piezoelectric
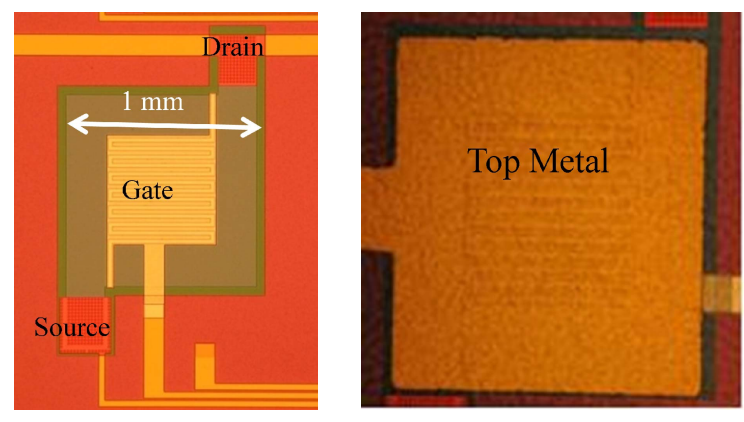

Figure 2: Enlarged picture of fabricated POSFET devices before (left) and after (right) deposition and processing of PVDF-TrFE piezoelectric polymer film.

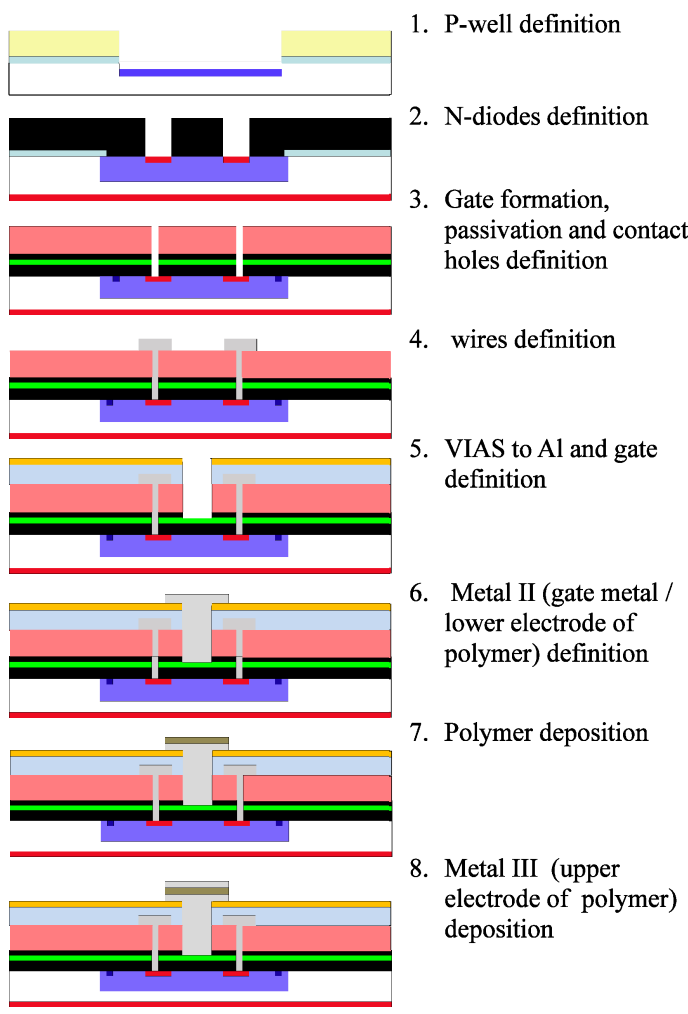

Figure 3: Fabrication steps of POSFET devices.

properties into PVDF and this becomes a critical issue if such a film is to be used in the sensors involving microfabrication. In addition to this, PVDF-TrFE demonstrates a higher level of piezoelectricity than PVDF [11]. It is for these reasons, that PVDF-TrFE polymer film is used in this work. The crystalline structure, and consequently the piezoelectric and pyroelectric properties of the polymer film depend on the molecular proportion $x$ $(0.6<x<0.85)$ of Vinyledene Fluoride in $\mathrm{P}\left(\mathrm{VDF}_{x^{-}}\right.$ 
$\left.\mathrm{TrFE}_{1-x}\right)$. Among various compositions of PVDF-TrFE, the one with $65 / 35$ weight ratio exhibits good ferroelectric response [11] and hence same is used in the POSFET tactile sensing devices.

\section{Polymer film deposition}

For a uniform response among various sensors, polymer films with uniform thickness are desired. The thickness of polymer depends on solution concentration, spinner's speed and time of spinning. Following a number of experiments performed on dummy silicon wafers (i.e. without any MOS device), $\sim 2.5 \mu \mathrm{m}$ thick PVDF-TrFE polymer film is deposited by spin coating a $10 \%$ solution prepared by stirring PVDF-TrFE pallets in RER500 solvent at $80^{\circ} \mathrm{C}$ for about 30 minutes - at $3000 \mathrm{rpm}$ for 30 seconds [12]. In fact, spin-coating is performed in three steps - each with different sets of spin rate and spin time. In first two steps both spin rate and spin time (500 rpm for $2 \mathrm{sec}$ followed by $600 \mathrm{rpm}$ for $8 \mathrm{sec}$ ) are kept smaller to allow the solution to spread uniformly over the whole wafer. In final spinning step, the spin rate is $3000 \mathrm{rpm}$ and spin time is 30 seconds. A higher concentrated solution, with higher spinning speed, can also be used to obtain $\sim 2.5$ $\mu m$ thick film. But it is found that the uniformity of polymer film thickness becomes poor with higher concentration [12].

\section{Annealing and Top Metallization}

To enhance the crystallization, to evaporate any left out solvent and to remove any local stress generated during deposition, the polymer film is annealed at $120^{\circ} \mathrm{C}$ for three hours. The temperature of $120^{\circ} \mathrm{C}$ is reached over a period of one hour and kept $120^{\circ} \mathrm{C}$ for three hours and then it is brought down to room temperature in one hour so as to release the stresses slowly. After annealing, the wafers are treated with HDMS, and then a $0.2 \mu \mathrm{m}$ top metal layer $(\mathrm{Au} / \mathrm{Cr})$ is vacuum deposited on films. HDMS treatment improves the adhesion of polymer and metal electrodes. The top metal was then patterned by standard wet etching procedures for $\mathrm{Au}$ and $\mathrm{Cr}$.

\section{Etching of Polymer Film}

The polymer needs to be etched, to clearly define the sensing area on the POSFET device and to gain access to the pads on the chip. Following a number of experiments involving wet and dry etch steps, on dummy silicon wafers (i.e. without any MOS device) [12], it was observed that dry etch with oxygen $(20 \mathrm{sccm})$ at $20 \mathrm{mBar}$ pressure and He (40 sssm) was sufficient to remove the polymer with a etching rate of $1 \mu \mathrm{m} / \mathrm{min}$ and hence same was used for etching the polymer film. Upper metal electrodes $(\mathrm{Au})$ acts as the mask for polymer during the dry etch step. The POSFET device after dry etching of the polymer is shown in Fig. 2. POSFET devices are diced and packaged, after the dry etching step.

\section{In-situ Poling of Polymer Film}

To improve the piezoelectric properties, the polymer film needs to be polarized. In the event when the sensors using piezoelectric polymers - are present on a chip having active devices, the poling process needs to be performed carefully to avoid damaging the devices and readout electronics. Polarization of PVDF-TrFE is generally done by applying a high electric field across its thickness. Resulting piezoelectric coefficient depends on the degree of polarization and hence on the strength of electric filed. Field strength of 80-100 V/ $\mu \mathrm{m}$ - which gets translated to 200-250 volts in present case - is required to polarize PVDF-TrFE films. With such a high voltage, it is challenging to polarize the polymer film, in situ, without damaging or altering the characteristics of underlying MOS device. To overcome this challenging step, all metals layers and substrate under the polymer film are grounded, before applying the voltage across the polymer film. Thermal poling [11] was performed at $85^{\circ} \mathrm{C}$, to reduce the voltage needed for polarization - 200 volts is finally used. A further care is taken by applying the voltage in four cumulative steps of 50 volts each [12]. In each step voltage is applied for 10 minutes followed by 5 minutes interval, during which, the two metal sides of polymer are shorted to neutralize any excess charge. Following these steps, the input and output characteristic of POSFET device are observed to be similar, both, before and after the polarization step, as can be noticed from Fig. 4.

The response of POSFET touch sensing device, to
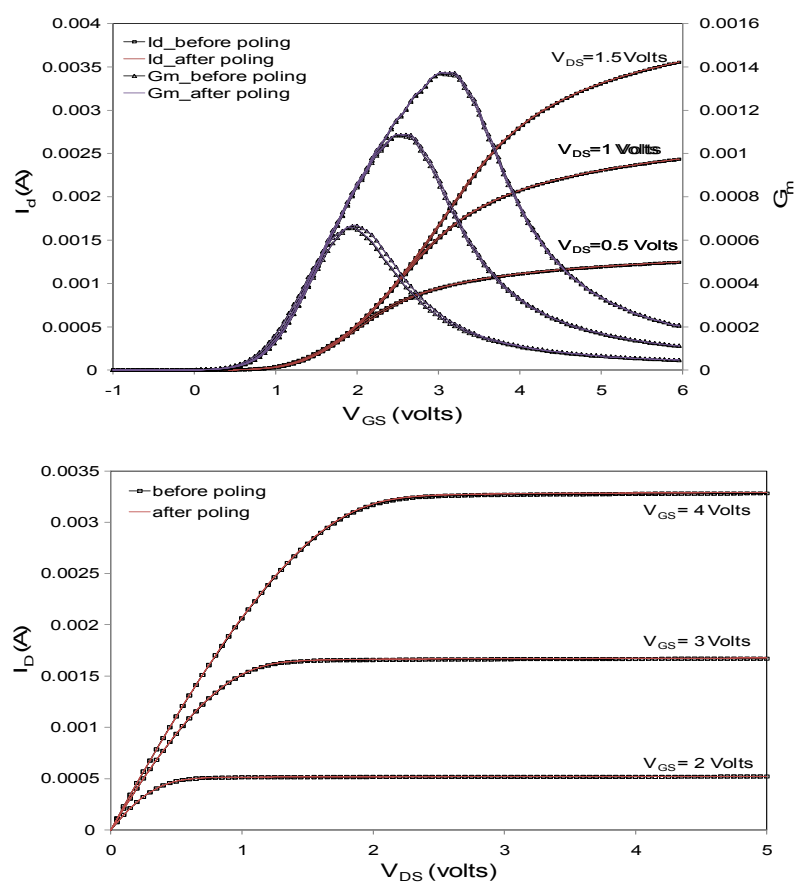

Figure 4: Input (top) and Output (below) characteristics of POSFET tactile sensing device before and after the poling. 


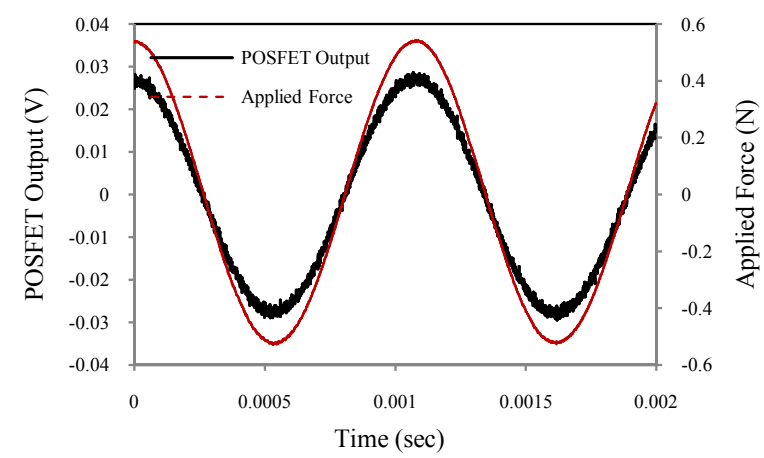

Figure 5: POSFET response to $925 \mathrm{~Hz}$ sinusoidal force.

áynamic numal forces, reconceú by conneciing ii in a source-follower circuit arrangement with floating gate, is shown in Fig. 5. A sinusoidal force $(925 \mathrm{~Hz})$ is applied in normal direction on top of device, as shown in Fig. 1. The experiment arrangement used to record the response of POSFET is explained elsewhere [13]. While applying force, the sensor was covered with a $300 \mu \mathrm{m}$ thin protective PDMS (Polydimethylsiloxane) rubber.

\section{CONCLUSION}

Design and Fabrication steps for implementing POSFET touch sensing devices are presented. Challenging issues like in situ poling of piezoelectric polymer film, without damaging or altering the characteristics of underlying MOS devices, have been successfully dealt with. The fabrication process is compatible with standard CMOS process.

Though primarily designed for robotic applications, these devices can be suitable for medical applications because of close impedance match between PVDF-TrFE and human tissues. POSFET touch sensing devices can also be used to obtain a conformal electronic surface by embedding them, as mechanically distinct and stiff sub circuit islands, in a soft and compliant polymer like PDMS and interconnect the islands with flexible and stretchable metallization.

\section{ACKNOWLEDGMENT}

This work is supported, in part; by the European Commission project RobotCub (IST-FP6-004370) and the Italian Ministry of Education University and Research, under the project PRIN 2007 "Tactile Sensing System for Humanoid Robots using Piezo-polymer-FET devices".

\section{REFERENCES}

[1] R. S. Dahiya and M. Valle, "Tactile Sensing for Robotic Applications," in Sensors, Focus on Tactile, Force and Stress Sensors, J. G. Rocha and S. Lanceros-Mendez, Eds. Vienna, Austria: I-Tech Education and Publishing, 2008, pp. 289-304.

[2] M. R. Cutkosky, R. D. Howe, and W. Provancher,
"Force and Tactile Sensors," in Springer Handbook of Robotics, B. Siciliano and K. Oussama, Eds. Berlin Heidelberg: Springer 2008, pp. 455-476.

[3] H. Takao, K. Sawada, and M. Ishida, "Monolithic Silicon Smart Tactile Image Sensor With Integrated Strain Sensor Array on Pneumatically Swollen SingleDiaphragm Structure," IEEE Transactions on Electron Devices, vol. 53, pp. 1250-1259, 2006.

[4] B. J. Kane, M. R. Cutkosky, and G. T. A. Kovacs, "A Traction Stress Sensor Array for Use in HighResolution Robotic Tactile Imaging," Journal of Microelectromechanical Sys., vol. 9, 425-434, 2000.

[5] T. Someya, T. Sekitani, S. Iba, Y. Kato, H. Kawaguchi, and T. Sakurai, " A Large-Area, Flexible Pressure Sensor Matrix with Organic Field-Effect Transistors for Artificial Skin Applications," PNAS, vol. 101, pp. 9966-9970, 2004.

[6] I. Manunza, A. Sulis, and A. Bonfiglio, "Pressure sensing by flexible, organic field effect transistors," Applied Physics Letters, vol. 89, pp. 143502, 2006.

[7] D. H. Kim, J. H. Ahn, H. S. Kim, K. J. Lee, T. H. Kim, C. J. Yu, R. G. Nuzzo, and J. A. Rogers, "Complementary Logic Gates and Ring Oscillators on Plastic Substrates by Use of Printed Ribbons of Single-Crystalline Silicon," IEEE Electron Devices Letters, vol. 29, pp. 73-76, 2008.

[8] R. S. Dahiya, M. Valle, and L. Lorenzelli, "SPICE Model of Lossy Piezoelectric Polymers," IEEE Transactions on Ultrasonics, Ferroelectrics and Frequency Control, vol. 56, pp. 387-396, 2009

[9] R. S. Johannson and I. Birznieks, "First Spikes in Ensembles of Human Tactile Afferents Code Complex Spatial Fingertip Events," Nature Neuroscience, vol. 7, pp. 170-177, 2008.

[10]S. Martinoia, N. Rosso, M. Grattarola, L. L., M. B., and M. Zen, "Development of ISFET array-based Microsystems for bioelectrochemical measurements of cell populations " Biosensors \& Bioelectronics, vol. 16, pp. 1043-1050, 2001.

[11]H. S. Nalwa, "Ferroelectric Polymers - Chemistry, Physics and Applications." New York: Marcel Dekker Inc., 1995.

[12]R. S. Dahiya, M. Valle, L. Lorenzelli, G. Metta, and S. Pedrotti, "Deposition Processing and Characterization of PVDF-TrFE Thin Films for Sensing Applications," IEEE Sensors 2008, Lecce, Italy, 2008.

[13]R. S. Dahiya, G. Metta, and M. Valle, "Development of Fingertip Tactile Sensing Chips for Humanoid Robots," 5th IEEE International Conference on Mechatronics, Malaga Spain, 2009 (in press).

\section{CONTACT}

* R. S. Dahiya, email: ravinder.dahiya@iit.it 